Klaus Peter Koch*, Julien Laigle Hoffmann, Lukas Jung, Eric Auer and Hartmut Schächinger

\title{
Psychometric test system for measuring cognitive reaction time
}

\author{
Verification of latency times and accuracy in visual stimuli caused by the test system
}

\begin{abstract}
For the assessment of human reaction time, a test environment was developed. This system consists of an embedded device with organic light-emitting diode (OLED) displays with push buttons for the combined presentation of visual stimulation and registration of the haptic human reaction. The test leader can define the test sequence with the aid of a graphical user interface (GUI) on a personal computer (PC). The validation of the system was proved by measuring the latency times of the whole system, which are conditioned by the specific hard- and software constellation. Through the investigation of the display's light radiation by a photodiode and the recorded current consumption, latency times and their variance were specified. In the fastest mode the system can reach an error limit of $60 \mu \mathrm{s}$.
\end{abstract}

Keywords: Reaction time measurement, cognitive performance assessment, OLED latency.

https://doi.org/10.1515/cdbme-2020-3147

\section{Introduction}

For assessment of the visual reaction time, a test environment is required that delivers consistent and reliable results without interference with the measurement system. Therefore, a hard- and software constellation for application in psychology laboratories was developed. Especially regarding the psychometric verification of the driver's aptitude, the scientific consensus finds that these test systems should be more standardized. [1] Furthermore, the quality factors of the test systems like reliability and statistical spread are often not documented. This missing information

\footnotetext{
*Corresponding author: Klaus Peter Koch, Technical department, Trier University of Applied Sciences, Trier, Germany, e-mail: koch@hochschule-trier.de

Julien Laigle Hoffmann, Lukas Jung, Eric Auer, Technical department, Trier University of Applied Sciences, Trier, Germany Hartmut Schächinger: Department of Clinical Psychophysiology, Trier University, Trier, Germany, e-mail: schaechi@uni-trier.de
}

could lead to misinterpretations. [1] Especially in cases where trials are not repeated very often, time accuracy is an important parameter for the stochastic evaluation of the results. Many of the commonly used measurement systems are based on software for PC systems. These are used on different systems with many variables, which affect unknown latency times, such as differing operation systems with unknown scheduling mechanisms, monitors and input devices. [2] The resulting inaccuracies of these constellation reach $20 \mathrm{~ms}$ with standard deviation of $2 \mathrm{~ms}$. [1] For this reason, the method here is based on an embedded system, where the software cycle is programmed near to the hardware, allowing more constant latency times to be achieved. Next to the development of such a system, the objectives of this work were to determine latency times, minimize their variances and describe how the stimuli are chronographically built up. An additional benefit of the designed system is the haptic quality of push buttons in combination with the direct sensomotoric relation between the directly picture on the button.

\section{Methods}

\subsection{Hardware concept}

The developed hardware, which will be handled by the test person, was defined for an open application field. It can be used as a stand-alone device. Therefore, the supply voltage can be obtained by an internal Li-Ion battery or by the power grid. Furthermore, a psychological test sequence database can be stored on a microSD memory card. Log files, which are generated during the test by the microcontroller (DSPIC33EP512GM710 from Microchip, $40 \mathrm{MHz}$ effective clock rate), are also stored on the memory card. For the presentation of visual stimuli, the industrial OLED Display ISF15ACP4 from NKK (96x64 pixels, 65536 colors) in push button combination was used, which is simultaneously able to represent pictures and register the test person's reaction i.e. 
pushing the button (see Fig. 1). The push button closes with a minimal force of $2 \mathrm{~N}(+/-0.5 \mathrm{~N})$.

Relevant digital and analog signals are accessible by a Sub-D port, which is connected to general-purpose input/output (GPIO) pins of the microcontroller and digital-to-analog converters (DACs). If a test sequence requires another type of stimuli, e.g. acoustic stimuli or trigger signals, external devices could be connected to the Sub-D port or an additional $3.5 \mathrm{~mm}$ phone jack.

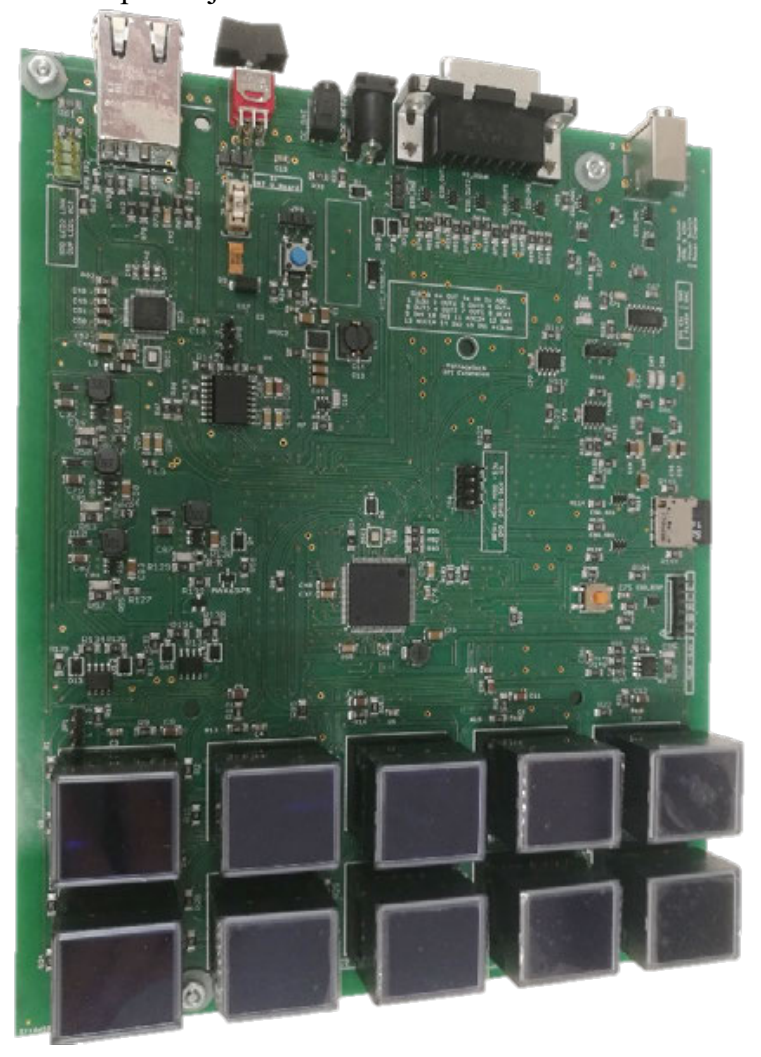

Figure 1: Picture of the test device's final hardware

\subsection{Software concept}

The software of the microcontroller is significant for the latency time, especially the OLED display control. In the first step, the microcontroller loads the random-access memory (RAM) with relevant data from the memory card. This information includes queue times between the visual stimuli, order of the used OLEDs, colors, brightness and so on.

A self-test of the OLED display avoids an incorrect or missing presentation of a stimulus. Therefore, every display is directed to flash on for a short time during the booting of the system. If the analog-to-digital converter (ADC) result of the microcontroller (see 2.3) does not confirm a current consumption for each single display, an error message will be saved in the log file of the respective trial.

For the display configuration, the image repetition rate lies in an interval between 96 and 117 fps. This variability is caused by huge tolerances of the OLED internal oscillators. Consequences for the latency times will be shown in chapter 3. The microcontroller software has two different modes which control different functions of the display driver SSD1331 to get the best performance:

Mode 1: Monochromatic color filling of one single display: In this mode, color changes are reached in the fastest way. However, the position of the first illuminated line is random.

Mode 2: Display of pictures. Each pixel is built up separately. Therefore, the configuration dataset of each pixel has to be transferred separately. The impact on latency time is shown in chapter 3 . In this mode it is guaranteed that the visual stimuli are the same for every display and it starts to build up the image from the first line at the top. This is done by filling the graphical ram during its boot-up time.

When the start time of the illumination has passed, the reaction time measurement begins. This is realized with a microcontroller's internal 16-bit and $5 \mathrm{MHz}$ pre-scaled timer module. After timer overflow, an interrupt service routine is called to increment a time step variable. The timer stops when the test person pushes a button. This is detected with a high priority interrupt on change command. It detects a level rise of any button signal input of the microcontroller and saves a time stamp. To prevent a queue of interrupt service routines by button bouncing, the buttons will be locked until each button signal level is checked. Summarized, in the first step the interrupt on change detects any keypress. After this, all buttons are locked until it is known which button was explicitly pushed.

\subsection{Test setup for latency time identification}

To record the chronological process of an image build up, an array of three QSB34CGR photodiodes (rise time $40 \mathrm{~ns}$ ) are arranged in a rectangle with the same dimensions as a display and with one photodiode above another (Fig. 2). To avoid measurement artifacts from other light sources, the array is shielded with a paper cylinder. These photodiodes are light sensitive in a visible electromagnetic spectrum between 400 and $1100 \mathrm{~nm}$. The light radiation-driven photocurrent leads to a detectable potential difference at the oscilloscope's internal impedance. Simultaneously, the current consumption of each 
display is recorded by a microcontroller's internal 12-bit ADC with a sampling timer interval of 225 ns. Every recording begins with a keypress, which delivers the trigger signal for timer, display command and oscilloscope. The display's internal memory is already loaded with color information.

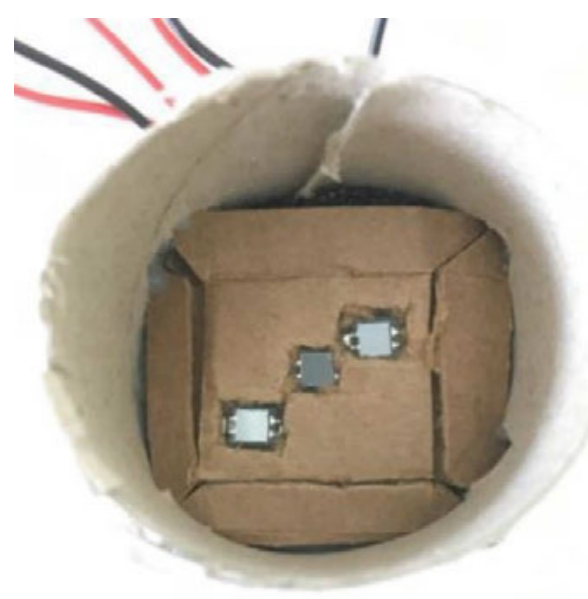

Figure 2: Light-shielded photodiode array

\section{Results}

Fig. 3 shows the oscilloscope time series of a display start sequence in mode 2. Signal $\mathrm{D}_{0}$ represents the timer steps. Marker 1) shows the end of the DisplayOn command and marker 2) shows the recording time of the ADC module to measure the supply current of the display. The three signals below come from the photodiodes.

$\square$ Green signal: Photocurrent of the bottom right photodiode

$\square \quad$ Blue signal: Photocurrent of the central photodiode

$\square \quad$ Yellow signal: Photocurrent of the top left photodiode

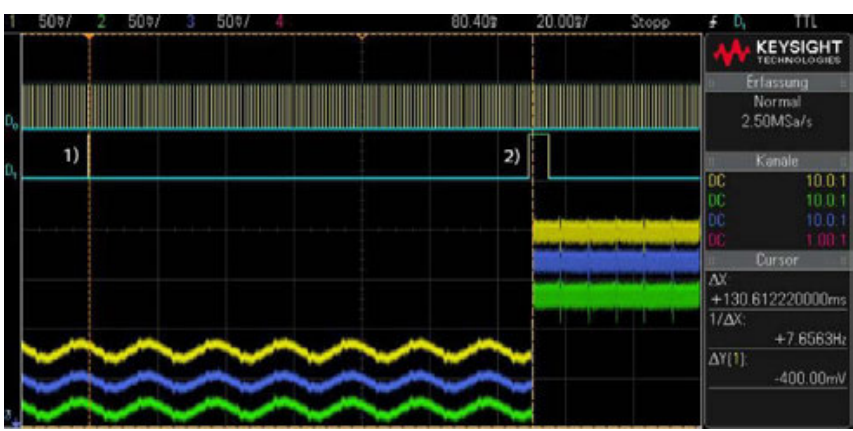

Figure 3: Time series for a display start

The photodiodes are placed $3 \mathrm{~cm}$ above the display. Measurements show a delay of $130 \mathrm{~ms}$ between DisplayOn command and start of illumination of the first row.
To investigate how a monochromatic image is chronographically built up in DisplayOn status, a DrawLine command is used, which loads the color value for each of the 64 rows. In this test, the photodiodes are directly

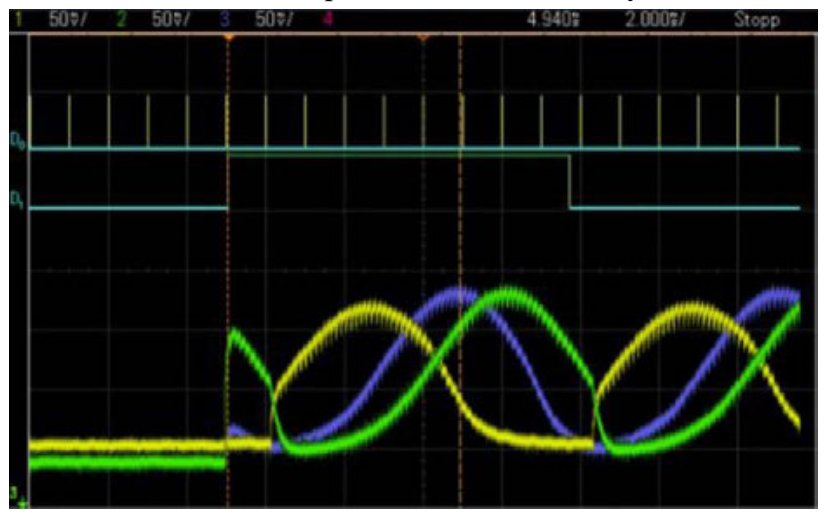

Figure 4: Image build-up beginning at actual pointer position

arranged above the display. In Fig. 4, the photocurrents illustrate the image build up. The oscillations are based on the distance of the photodiode and the actual position of the image build up. Many repetitions showed that the first illumination in this display-controlling mode depends on the actual pointer position of the graphical memory. Hence, it is not predictable where the first visual stimulus on a display begins. As an example, in Figure 4 the first photodiode, which detects light radiation, is positioned in the bottom right (green signal).

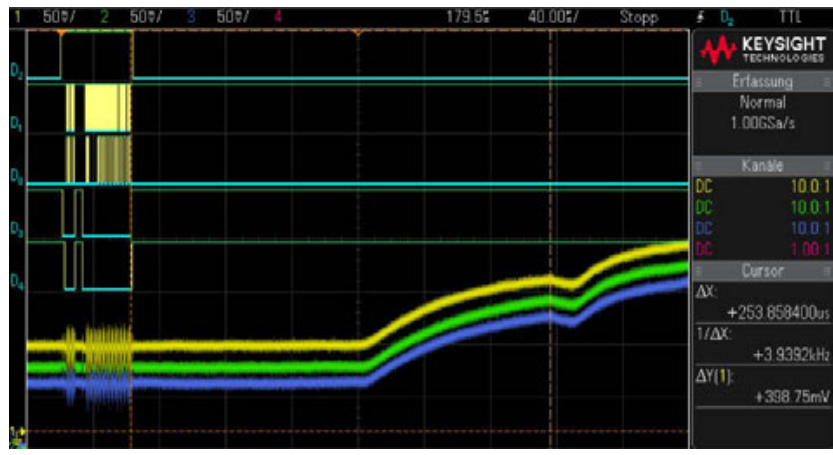

Figure 5: Image build-up in a row

Fig. 5 depicts how long it takes until a monochromatic visual stimulus is noticeable, if a display is already turned on in mode 1. The used command SetColor in this case is specified for filling a whole screen by sending only 2 bytes. This corresponds to a transmission time of $43.6 \mu \mathrm{s}$. After the data sequence has been received, internal memory management of the display leads to a first light detection after $200 \mu \mathrm{s}$. The time until a first row illuminates completely takes on average $260 \mu \mathrm{s}$. The equal distribution of the deviations from the mean value resulting from the time 
discretization. These error limits are $+/-60 \mu \mathrm{s}$. This has been determined by a measurement series with 50 trials.

The push bottom detection with separate microcontroller inputs was programmed in millisecond steps. As a consequence, in the worst case, the detected reaction time ignores a whole millisecond and on average $500 \mu$ s with the error limits of $+/-500 \mu \mathrm{s}$. The programming of the timer can reduce this latency time.

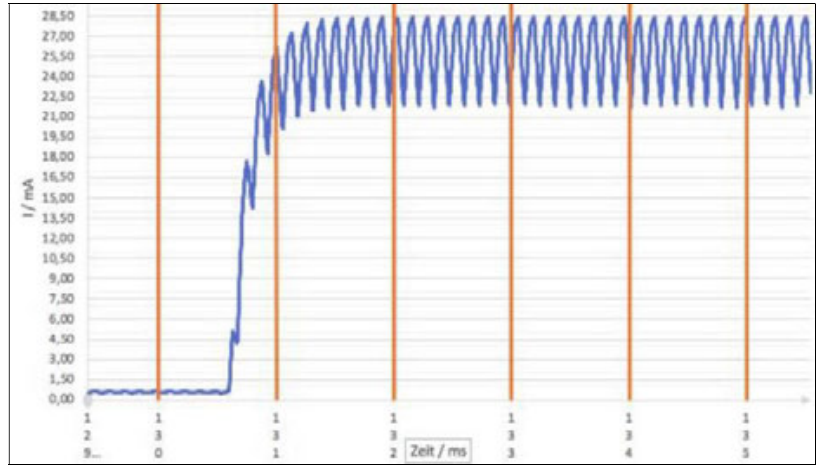

Figure 6: Current consumption on display start

If an individual stimulus uses images instead of single colors, the accelerated display driver functions are not utilizable. Therefore, in mode 2, raw pixel information needs to be loaded in the graphical memory, which takes $40 \mathrm{~ms}$. If the display is already displaying something and the information is received, it can occur that the new image is overlaid with an old image which is still displayed. To avoid this effect and to achieve repetitive stimuli conditions, the display is loaded during its boot phase of $130 \mathrm{~ms}$, which is enough time to acquire all data. After boot-up, the display starts to show its first line always at the top. The variation of the boot phase to start the build-up process is in the limits of $+/-900 \mu \mathrm{s}$. This process is the same as shown in Fig. 3. The time until a whole picture is displayed on the 64 rows takes $8 \mathrm{~ms}$.

Fig. 6 shows the current consumption time series of a display start process. It is observable, how the image is slowly built up between $130 \mathrm{~ms}$ and $131 \mathrm{~ms}$ from row to row. But it is not useful for verification of the latency time directly, because information on the stimulus presence is missing. Table 1 shows a compilation of important results.

Table 1: Verified time intervals

\begin{tabular}{|c|c|c|}
\hline Event & Mean values & Error limits \\
\hline $\begin{array}{l}\text { Display turn on (Mode 2) } \\
\text { incl. transmission time for a } \\
\text { complete picture }\end{array}$ & $130 \mathrm{~ms}$ & $+/-900 \mu \mathrm{s}$ \\
\hline
\end{tabular}

\begin{tabular}{lll}
\hline Event & Mean values & Error limits \\
\hline $\begin{array}{l}\text { Color filling first row active } \\
\text { (Mode1) }\end{array}$ & $260 \mu \mathrm{s}$ & $+/-60 \mu \mathrm{s}$ \\
$\begin{array}{l}\text { Color filling first pixel active } \\
\text { (Mode1) }\end{array}$ & $200 \mu \mathrm{s}$ & $+/-60 \mu \mathrm{s}$ \\
$\begin{array}{l}\text { Color filling whole display active } \\
\text { Transmission time }\end{array}$ & $8 \mathrm{~ms}$ & $+/-800 \mu \mathrm{s}$ \\
$\begin{array}{l}\text { SetColor (Mode1) } \\
\begin{array}{l}\text { Transmission time } \\
\text { SetPixel for all Pixels (Mode 2) }\end{array}\end{array}$ & $43.6 \mu \mathrm{ms}$ & \\
$\begin{array}{l}\text { Inaccuracy in reaction time based } \\
\text { on PC-measurement system [1] }\end{array}$ & $20.7 \mathrm{~ms}$ & $\begin{array}{l}2 \text { ms } \\
\text { (standard } \\
\end{array}$ \\
& & deviation) \\
\hline
\end{tabular}

\section{Conclusions}

This work was intended to create a test environment with a defined time measurement accuracy. It was shown that the introduced hard- and software constellation can present fast visual stimuli within $200 \mu \mathrm{s}-320 \mu$ s for the first line single colors. The presentation of new pictures lasts at least between $129.1 \mathrm{~ms}$ and $130.9 \mathrm{~ms}$ for the first stimulus. It takes an additional $8 \mathrm{~ms}$ to build up the whole picture. The test leader has to decide when a stimulus is said as occurred. This could be the imagination of a first pixel, first row, or a whole display. As the mean value of the delay is known the error limits for single color and picture are $+/-60 \mu \mathrm{s}$ and $+/-$ $900 \mu$ s, respectively.

The inaccuracy of the reaction detection with up to $+/-0.5 \mathrm{~ms}$ causes a relevant result derivation. By reconfiguring the timer setup, the resolution will be increased by adaptation of the timer programming. This improvement could be relevant for test scenarios without a lot of repetitions, which are purposed to correct the stochastic errors. Future studies will also investigate how other communication ports can be included in the test leader's workflow.

\section{References}

[1] Poschadel S., Falkensteil M.: Aach T. Testverfahren zur psychometrischen Leistungsprüfung der Fahreignung, Berichte der Bundesanstalt für Straßenwesen: Mensch und Sicherheit; Heft M 2032009.

[2] Reimers S., Stewart, N., Adobe Flash as a medium for online experimentation: A test of reaction time capabilities, Behavior Research Methods 2007: 39(3), 365-37 